\title{
Uso de catéter venoso central de inserción periférica en pediatría
}

\author{
PAULA FAJURI M. ${ }^{1,4}$, PAOLA PINO A. ${ }^{2}$, ANDRÉS CASTILLO M. ${ }^{3,4}$ \\ 1. Enfermera Matrona. \\ 2. Enfermera. Servicio de Pediatría. Hospital Clínico Pontificia Universidad Católica de Chile. \\ 3. Médico. División de Pediatría. \\ 4. Unidad de Paciente Crítico Pediátrico. Hospital Clínico Pontificia Universidad Católica de Chile.
}

\section{ABSTRACT \\ Central venous catether for peripheral insertion in pediatrics}

Introduction: Central venous catheter of peripheral insertion (PICC) can stay installed from several days up to months without removal. It allows the administration of extreme $\mathrm{pH}$ and osmolarity solutions, irritating and/or vesicant drugs, parenteral nutrition and other medications for prolonged periods in patients with complicated peripheral venous access. Objective: To describe the experience with PICCs in hospitalized pediatric. Patients and Methods: Observational and descriptive study on 337 patients hospitalized in the Pediatrics Service of the Clinical Hospital of Catholic University of Chile between 2001 and 2011, who fulfilled the inclusion criteria and had a PICCs installed by trained nurses. Results: The patients' average age was of 36 months. Main indication for installing PICC was prolonged antibiotic therapy, in $67.1 \%$ of cases. The most widely used venous route was the upper limb, in $52.2 \%$. The mean average time in which the PICC remained in the body was of 9 days, ranging between 1 and 90 days. The main cause for PICC withdrawal was the end of therapy in $75.3 \%$. The observed complications were: occlusion and catheter associated infection in $8.9 \%$ and $2.9 \%$ respectively. Conclusion: PICC is an excellent alternative for prolonged intravenous therapy; but, it is very important to keep on a team of well-trained nurses, both in the insertion as well in the maintenance of the PICC during the time it remains in the body.

(Key words: Central catheter of peripheral insertion, PICC, percutaneous catheter, pediatrics).

Rev Chil Pediatr 2012; 83 (4): 352-357

\section{RESUMEN}

Introducción: El catéter venoso central de inserción periférica (PICC) puede permanecer desde días hasta meses instalado sin necesidad de recambio; permitiendo la administración de soluciones con pH y osmolaridad extremas, medicamentos irritantes y/o vesicantes, nutrición parenteral u otros medicamentos por tiempo prolongado en pacientes con accesos venosos periféricos difíciles. Objetivo: Describir la experiencia del uso de PICCs en pacientes pediátricos hospitalizados. Pacientes y Método: Estudio observacional y descriptivo, donde se hizo un seguimiento a 337 pacientes con PICCs instalados por enfermeras capacitadas, en el Servicio de Pediatría del Hospital Clínico de la Pontificia Universidad Católica de Chile entre los años 2001 y 2011, que

Trabajo recibido el 02 de agosto de 2011, devuelto para corregir el 27 de noviembre de 2011, segunda versión el 30 de enero de 2012, aceptado para publicación el 12 de marzo de 2012.

Correspondencia a:

Paula Fajuri M.

E-mail: pfajuri@med.puc.cl 
cumplieron con los criterios de inclusión. Resultados: La edad de los pacientes presentó una mediana de 36 meses. La principal indicación para la instalación del PICC fue la terapia antibiótica prolongada en el 67,1\%. El acceso venoso más utilizado fue la extremidad superior en un 52,2\%. El promedio de días de permanencia del catéter presentó una mediana de 9 días con un rango entre 1 y 90 días. El principal motivo para el retiro del PICC fue la finalización del tratamiento en el 75,3\%. Las complicaciones presentadas fueron: oclusión e infección asociada al catéter con un $8,9 \%$ y $2,9 \%$, respectivamente. Conclusión: El PICC es una excelente alternativa para la terapia endovenosa por períodos prolongados; sin embargo, es muy importante mantener un equipo de enfermería capacitado, tanto en la inserción como en la mantención del PICC durante su permanencia.

(Palabras clave: Catéter central de inserción periférica, PICC, catéter percutáneo, pediatría).

Rev Chil Pediatr 2012; 83 (4): 352-357

\section{Introducción}

En el pasado, los accesos venosos centrales eran instalados en pabellón, bajo anestesia general $^{1}$, siendo un procedimiento no exento de riesgos y asociado a eventos adversos descritos en la literatura ${ }^{2}$.

El catéter central de inserción periférica (PICC) es instalado por enfermeras calificadas, su extremo distal se localiza en la vena cava superior, pudiendo permanecer desde días hasta meses instalado sin necesidad de recambio $^{3}$. Éste catéter permite la administración de soluciones y medicamentos con $\mathrm{pH}$ extremo, siendo irritantes aquellos cuyo $\mathrm{pH}$ es mayor a 7,45 y vesicantes cuyo $\mathrm{pH}$ es menor a 7,35 (tabla 1). También permite la administración de soluciones y medicamentos hiperosmolares, es decir $\geq 350 \mathrm{mOsm} / \mathrm{L}$ (tabla 2); y otras soluciones y medicamentos por tiempo prolongado en pacientes con accesos venosos periféricos difíciles ${ }^{4-6}$.

El Centro de Control y Prevención de Enfermedades (CDC) en su Guía de Recomendaciones para la prevención de infecciones relacionadas con accesos vasculares 2011, promueve el uso de PICCs cuando la terapia intravenosa excede los 6 días de duración ${ }^{7}$.

Las ventajas de éste tipo de catéter incluyen: una marcada reducción en el trauma relacionado con el procedimiento (neumotórax, hemotórax o punción arterial accidental), menor riesgo de sangramiento y de infección asociada al catéter, instalación más económica y mantención menos dificultosa ${ }^{8}$, brindando también mayor comodidad al paciente.

Las complicaciones asociadas al uso de este catéter pueden ser tempranas o tardías, entre las tempranas se encuentran: ubicación inadecuada, migración o fractura del catéter; los eventos tardíos incluyen: migración, fractura, infección, trombosis venosa, disfunción del catéter y flebitis ${ }^{8}$.

En Chile, este tipo de catéter se utiliza en servicios de neonatología y pediatría en el sector público y privado, encontrando en la literatura sólo un estudio descriptivo del uso de PICCs en neonatología en un hospital docente

Tabla 1. pH de soluciones y medicamentos utilizados

\begin{tabular}{|lc|}
\hline Solución o medicamento & pH \\
\hline Aciclovir & $10,5-11,6$ \\
\hline Albumina & $6,4-7,5$ \\
\hline Diazepam & $6,2-6,9$ \\
\hline Dopamina & $2,5-4,5$ \\
\hline Epinefrina & $2,5-5,0$ \\
\hline Fenobarbital & $8,5-10,5$ \\
\hline Ganciclovir & 11,0 \\
\hline Gluconato de calcio 10\% & $6,0-8,2$ \\
\hline Inmunoglobulina & $4,0-7,2$ \\
\hline Levofloxacino & $3,8-5,8$ \\
\hline Metilprednisolona & $7,0-8,0$ \\
\hline Vancomicina & 2,4 \\
\hline
\end{tabular}

Tabla 2. Osmolaridad de soluciones y medicamentos utilizados

\begin{tabular}{|lr|}
\hline Solución o medicamento & \multicolumn{1}{c|}{ Concentración } \\
\hline Suero glucosado al 10\% & $505 \mathrm{mOsm} / \mathrm{L}$ \\
\hline Nutrición parenteral total & $1500-3500 \mathrm{mOsm} / \mathrm{L}$ \\
\hline Nutrición parenteral parcial & $750-1500 \mathrm{mOsm} / \mathrm{L}$ \\
\hline Manitol & $274-1372 \mathrm{mOsm} / \mathrm{L}$ \\
\hline
\end{tabular}


de Valdivia ${ }^{9}$. Sin embargo, al revisar bases de datos de revistas nacionales, no se han encontrado publicaciones relacionadas.

El objetivo del estudio es describir la experiencia del uso de PICCs en pacientes pediátricos hospitalizados en un Servicio de Pediatría de un hospital docente de Santiago, entre los años 2001 y 2011.

\section{Pacientes y Método}

Se realizó un estudio observacional y descriptivo, donde se hizo un seguimiento de todos los PICCs instalados en el Servicio de Pediatría del Hospital Clínico de la Pontificia Universidad Católica de Chile en el período comprendido entre agosto del año 2001 y mayo del año 2011, correspondiendo a un universo de 357 catéteres. La instalación de los catéteres fue realizada por enfermeras del mismo servicio capacitadas para dicho procedimiento, bajo sedación moderada a profunda supervisada por un médico intensivista pediátrico.

Se excluyeron de este análisis los PICCs de pacientes que fueron trasladados a otro centro hospitalario u otro servicio el mismo día de la instalación del catéter $(\mathrm{n}=20)$.

Las variables estudiadas fueron: sexo, edad y diagnóstico de ingreso del paciente; indicación para la instalación, acceso venoso utilizado, calibre del catéter y número de lúmenes, días de permanencia, motivo de retiro del PICC y complicaciones asociadas al uso del catéter.

Los datos fueron recolectados el mismo día de la instalación del catéter y una vez que éste fue retirado, utilizando la hoja de registro diario de enfermería, el libro de ingreso del servicio y el seguimiento interno de infecciones asociadas a la atención de salud (IAAS).

Para el análisis de los datos se utilizó el Programa SPSS 15.0. Se realizaron medidas de tendencia central y dispersión, y análisis de frecuencia.

\section{Resultados}

Durante el período de seguimiento fueron estudiados 337 pacientes que cumplieron con los criterios de inclusión. De ellos, $51 \%$ es de sexo masculino. La edad promedio de los niños es 55,53 $\pm 54,6$ meses, con una mediana de 36 meses (rango entre 0,06 y 240 meses). El diagnóstico de ingreso más frecuente de los pacientes es la enfermedad respiratoria (tabla 3 ).

La principal indicación para la instalación del catéter fue la necesidad de tratamiento antibiótico por más de 5 días (tabla 4). En la categoría otras indicaciones se incluyen: uso de sedación, drogas vasoactivas, suero glucosado al $20 \%$ y otros medicamentos irritantes para ser administrados por vía venosa periférica.

El acceso venoso más utilizado correspondió a la extremidad superior $(52,2 \%)$, seguido del acceso yugular $(41,2 \%)$ y un $6,6 \%$ en otros

Tabla 3. Distribución porcentual según diagnóstico de ingreso

\begin{tabular}{|lcc|}
\hline Diagnóstico de ingreso & Frecuencia & Porcentaje \\
\hline $\begin{array}{l}\text { Enfermedad respiratoria } \\
\text { Enfermedad cardiovascular y } \\
\text { cirugía cardíaca }\end{array}$ & 92 & $27,3 \%$ \\
\hline $\begin{array}{l}\text { Enfermedad gastrointestinal } \\
\text { y cirugía abdominal }\end{array}$ & 40 & $22 \%$ \\
$\begin{array}{l}\text { Enfermedad neurológica y } \\
\text { neuroquirúrgica }\end{array}$ & 36 & $11,9 \%$ \\
\hline $\begin{array}{l}\text { Enfermedad osteoarticular y } \\
\text { de partes blandas }\end{array}$ & 31 & $9,7 \%$ \\
\hline $\begin{array}{l}\text { Enfermedad renal } \\
\text { Otros diagnósticos }\end{array}$ & 31 & $9,2 \%$ \\
\hline Total & 33 & $9,8 \%$ \\
\hline
\end{tabular}

Tabla 4. Distribución porcentual según indicación para la instalación del PICC

\begin{tabular}{|lcc|}
\hline Indicación & Frecuencia & Porcentaje \\
\hline Antibiótico & 226 & $67,1 \%$ \\
\hline Acceso seguro & 24 & $7,1 \%$ \\
\hline $\begin{array}{l}\text { Antibióticos y nutrición } \\
\text { parenteral }\end{array}$ & 22 & $6,5 \%$ \\
\hline Nutrición parenteral & 14 & $4,2 \%$ \\
\hline $\begin{array}{l}\text { Fenitoína } \\
\text { Antibiótico y otros medica- } \\
\text { mentos }\end{array}$ & 12 & $3,6 \%$ \\
$\begin{array}{l}\text { Nutrición parenteral y otros } \\
\text { medicamentos }\end{array}$ & 10 & $2,9 \%$ \\
\hline Otras indicaciones & 3 & $0,9 \%$ \\
\hline Total & 26 & $7,7 \%$ \\
\hline
\end{tabular}


lugares (safena, poplítea, temporal). El 97,9\% de los PICCs instalados fue de un lumen 2,8 french y el resto correspondió a doble lumen 5 french. El promedio de días de permanencia fue 12,9 $\pm 13,1$ días, con una mediana de 9 días (rango entre 1 y 90 días).

Los motivos para el retiro del catéter se presentan en la tabla 5. En la categoría otros motivos se incluyen: desplazamiento, ruptura, ubicación inadecuada, trombosis, flebitis, retiro accidental, filtración e instalación de catéter venoso central. Un $4,5 \%$ de los pacientes fue trasladado a otro servicio o institución con el catéter instalado.

Una de las complicaciones asociadas al uso del catéter en el estudio, fue la oclusión del lumen del catéter. De los 30 pacientes que presentaron esta complicación, un 30\% estaba recibiendo Fenitoína endovenosa a través del

Tabla 5. Distribución porcentual según motivo de retiro del PICC

\begin{tabular}{|lcc|}
\hline Motivo de retiro & Frecuencia & Porcentaje \\
\hline Fin de tratamiento & 254 & $75,3 \%$ \\
\hline Oclusión del lumen & 30 & $8,9 \%$ \\
\hline Pacientes trasladados & 15 & $4,5 \%$ \\
\hline Fallece & 10 & $3,0 \%$ \\
\hline Infección asociada al catéter & 10 & $2,9 \%$ \\
\hline Otros motivos & 18 & $5,3 \%$ \\
\hline Total & 337 & 100 \\
\hline
\end{tabular}

PICC, entre los años 2002 y 2003. El 23,3\% se ocluyó por otros motivos (desconexión, reflujo, baja velocidad de infusión, desconocimiento del manejo del catéter), y en $46,7 \%$ de los casos no se identificó la causa de la oclusión. Dentro de otras complicaciones, se encontró que $2,9 \%(\mathrm{n}=10)$ de los pacientes presentó infección del torrente sanguíneo asociada al catéter, lo que corresponde a 2,4 x 1000 días catéter. Las características de los pacientes que presentaron infección se presentan en la tabla 6 .

\section{Discusión}

Este es el primer estudio desarrollado en Chile, específicamente en el área pediátrica que muestra la experiencia en el uso de PICCs en pacientes pediátricos, dado que, como se aprecia en la literatura, la mayor experiencia se encuentra en el área de neonatología, donde hay bastantes estudios al respecto con resultados favorables ${ }^{2,9-11}$.

Las características de los participantes son bastante distintas a las encontradas en otros estudios donde la mayoría son neonatos ${ }^{2,9-11}$ y sólo tres estudios son en pacientes menores de 18 años $^{5,12,13}$ y dos estudios en pacientes adul$\operatorname{tos}^{4,5}$.

La indicación de terapia antibiótica prolongada sigue siendo la principal indicación para la instalación de un PICC, lo cual concuerda con otros estudios donde más del $50 \%$ de ca-

Tabla 6. Características de los pacientes con PICCs que presentaron infección

\begin{tabular}{|c|c|c|c|c|c|c|}
\hline Sexo & Edad & Diagnóstico & Indicación & Acceso & Días & Germen \\
\hline $\mathrm{F}$ & 9 & Enfermedad gastrointestinal y cirugía abdominal & NPT & Yugular & 7 & S. Aureus \\
\hline M & 108 & Enfermedad gastrointestinal y cirugía abdominal & NPT-ATB & Yugular & 7 & S. Aureus \\
\hline $\mathrm{F}$ & 72 & Enfermedad gastrointestinal y cirugía abdominal & ATB & Yugular & 8 & E. Coag $(-)$ \\
\hline M & 0,3 & Enfermedad cardiovascular y cirugía cardíaca & ATB & Yugular & 11 & E. Asbueriae \\
\hline $\mathrm{F}$ & 12 & Enfermedad gastrointestinal y cirugía abdominal & Otras & Yugular & 17 & S. Aureus \\
\hline $\mathrm{M}$ & 96 & Otras & ATB & Brazo & 17 & E. Maltofilia \\
\hline $\mathrm{F}$ & 21 & Enfermedad neurológica y neuroquirúrgica & ATB & Brazo & 25 & C. Parapsilosis \\
\hline $\mathrm{F}$ & 84 & Otras & ATB-NPT & Brazo & 33 & E. Coag $(-)$ \\
\hline M & 3 & Enfermedad respiratoria & Otras & Yugular & 56 & E. Cloacae \\
\hline M & 9 & Enfermedad cardiovascular y cirugía cardíaca & ATB & Safena & 70 & C. Tropicalis \\
\hline
\end{tabular}


téteres fueron instalados para este efecto. No obstante la nutrición parenteral y la administración de quimioterapia también son motivo de instalación de un PICC; ya que también son terapias endovenosas prolongadas, con medicamentos irritantes y vesicantes que no deben ser administrados por vía venosa periférica ${ }^{4,5,10,12}$.

Con respecto al acceso venoso utilizado, la literatura describe como lugar más frecuente para la instalación de los PICCs, las venas ubicadas en las extremidades superiores; ya que presentan una estructura y anatomía bien definida ${ }^{4,10,11,13}$. No obstante, en este estudio el acceso yugular cobra casi igual importancia con $41,2 \%$ vs $52,2 \%$ de acceso en los brazos lo que puede estar determinado en este grupo de pacientes porque la indicación de un PICC algunas veces es tardía, siendo difícil acceder a las venas de los brazos pues ya se encuentran multipuncionadas.

La permanencia de los PICC en el presente estudio es muy similar a la observada en otros estudios donde el promedio de días fue de 13 a 24 días, con un rango entre 1 y 155 días ${ }^{4,5,13}$; destacando que muchas veces cuando se suspende la terapia antibiótica, el catéter se mantiene como acceso venoso seguro con el fin de evitar la multipunción.

Al investigar el motivo de retiro del catéter, en la gran mayoría fue por fin de tratamiento. Otra causa importante y descrita en la literatura es la oclusión del lumen del catéter, representando en este estudio un $8,9 \%$, porcentaje más bajo que en la literatura, donde el reporte va desde un $10 \%$ a un $20 \%^{2,4,12}$. Lo anterior, es probablemente explicado porque en la institución desde el año 2009 se han desarrollado protocolos de inserción, manejo y retiro de los PICCs, lo que ha optimizado su manejo.

La administración de fenitoína endovenosa fue causa de retiro en $30 \%$ de los catéteres ocluidos durante los años 2002 y 2003, medicamento descrito en la literatura como factor importante en la oclusión, ya que forma cristales dentro del catéter durante su administración al igual que el diazepam endovenoso ${ }^{2}$. Posterior a ese período, sólo se describe 1 catéter ocluido por esta causa; ya que se realizó una capacitación dirigida a todas las enferme- ras asistenciales del servicio para la administración adecuada de este medicamento.

Con respecto al retiro del catéter por infección, se reporta como causa para este estudio en $2,9 \%$, correspondiendo a una tasa de $2,4 \times$ 1000 días catéter, menor incluso que la tasa ministerial, que corresponde a un 3,1 x 1000 días catéter, tasa que analiza infección del torrente sanguíneo asociada a catéter venoso central (CVC) sin discriminar entre PICC y CVC. Reportes de otros estudios dan a conocer tasas de infección que fluctúan entre $2 \%$ y $21 \%{ }^{11-13,15,16}$; entre los factores asociados a esta complicación se encuentran: técnica aséptica inadecuada al momento de la instalación, excesiva manipulación del catéter, susceptibilidad del paciente según su patología de base, y uso de nutrición parenteral ${ }^{15}$.

Entre las fortalezas del estudio se encuentran el número de pacientes incluidos, el período de seguimiento y la escasa pérdida de seguimiento en pacientes con PICC.

Entre las limitaciones del presente estudio se encuentran la falta de seguimiento a los pacientes trasladados a otras instituciones con catéter y algunas variables que no fueron descritas como, el número de intentos para la instalación, la ubicación radiográfica exacta del catéter y las causas de oclusión del catéter en 21 pacientes.

\section{Conclusión}

El PICC es un método eficaz y seguro en pacientes pediátricos, constituyendo una excelente alternativa para la administración de soluciones y medicamentos irritantes, vesicantes e hiperosmolares, y otros medicamentos por períodos prolongados. Es importante mantener un equipo de enfermería capacitado, tanto en la inserción como en la mantención del PICC durante su permanencia, medida que podría evitar algunas de las complicaciones más frecuentes como la oclusión del lumen y la infección asociada al catéter ${ }^{17}$.

Esta investigación es la primera que se realiza en el Servicio de Pediatría en relación al uso de PICCs, por lo que constituye un gran aporte al manejo de los pacientes y desarrollo del servicio. Sin perjuicio de lo anterior, sería 
un gran aporte estudiar otros factores que influyen en la permanencia de los PICCs, entre los que se encuentran, evaluación de la técnica de inserción del catéter por parte de las enfermeras, conocimiento de éstas acerca del manejo de éste tipo de catéter, y satisfacción usuaria en pacientes y sus familias.

\section{Agradecimientos}

Al equipo de enfermeras que participó en la instalación de los PICCs.

Al Dr. Jaime Cerda, Especialista en Salud Pública. Departamento de Salud Pública PUC; quien realizó el análisis estadístico de los datos.

A la PhD María Teresa Urrutia, Profesora Asociada. Escuela de Enfermería PUC; quien supervisó la redacción del manuscrito.

\section{Referencias}

1.- Crowley J: Vascular access. Techniques in Vascular and Interventional. Radiology 2003; 6 (4): 176-81.

2.- Tomazi A, Chollopetz M: Adverse events related to the use of central venous catheter in hospitalized newborns. Rev. Latino-Am. Enfermagem 2010; 18 (2): 196-202.

3.- Periard D, Monney P, Waeber G, et al: Randomized controlled trial of peripherally inserted central catheter versus peripheral catheter for middle duration inhospital intravenous therapy. J Thromb Haemost 2008; 6: 1281-8.

4.- Gasparotto G, Braga J: The use of peripherally inserted central catheter (PICC) in the hospital environment. Rev. Latino-Am. Enfermagem 2010; 18 (6): 1131-7.

5.- Tolomeo C, Mackey W: Peripherally inserted central catheters (PICCs) in the CF population: One center's experience. Pediatric Nursing 2003; 29 (5): 355-9.

6.- Eloy L, Tavares $M$, Gomes $M$, Lenz $N$, Mantegassi $R$, Nascimento S: Terapia Intravenosa utilizando Cateter Central de Inserçào periférica (CCIP). 2011. Sào Paulo,
Brasil: Iàtria.

7.- O'Grady N, Alexander M, Burns L, et al: Guidelines for the prevention of intravascular catheter-related infections. Am J Infect Control 2011; 39 (4 Suppl 1): 1-34.

8.- Amerasekera S, Jones C, Patel R, Cleasby M: Imaging of the complications of peripherally inserted central venous catheters. Clin Radiol 2009; 64: 832-40.

9.- Barría M, Sanyander G: Cateterismo venoso central de inserción periférica en recién nacidos de cuidado intensivo. Rev Chil Pediatr 2006; 77 (2): 139-46.

10.- Gomes A, Nascimento M, Antunes J, Araújo M: El catéter percutáneo en la unidad de cuidados intensivos neonatales: Una tecnología del cuidado de enfermería. Enfermería Global 2010; 19: 1-13.

11.- Romaniello H: Incidencia de extravasación del líquido de perfusión por el uso de catéteres percutáneos. Arch Argent Pediatr 2005; 103 (1): 31-5.

12.- Bui S, Babre F, Hauchecorne S, et al: Intravenous peripherally central catheters for antibiotic therapy in children with cystic fibrosis. J Cystic Fibrosis 2009; 8: 326-31.

13.- Dubois J, Rypens F, Garel L, David M, Lacroix J, Gauvin F: Incidence of deep vein thrombosis related to peripherally inserted central catheters in children and adolescents. CMAJ 2007; 177 (10): 1185-90.

14.- Ministerio de Salud: Informe de vigilancia epidemiológica de Infecciones intrahospitalarias. Vigilancia Infecciones Intrahospitalarias. 2007. Disponible en: http:// www.redsalud.gov.cl/portal/url/item/73627aa2edca037 4e04001011 f01734d.pdf.

15.- Corrèa V, Secoli R: Complicaçòes acerca do catéter venoso central de inserçào periférica (PICC). Cienc Cuid Saude 2007; 6 (2): 252-60.

16.- Bonet A, Grau $T$ y el Grupo de Trabajo de Metabolismo y Nutrición de la Sociedad Española de Medicina Intensiva Critica y Unidades Coronarias: Estudio multicéntrico de incidencia de las complicaciones de la nutrición parenteral total en el paciente grave. Estudio ICOMEP $1^{\text {a }}$ parte. Nutr Hosp 2005; 20 (4): 268-77.

17.- Lourenco S, Ohara C: Conocimiento de los enfermeros sobre la técnica de inserción del catéter central de inserción periférica en recién nacidos. Rev Latino-Am Enfermagem 2010; 18 (2): 1-8. 